Original Research Article

\title{
Accuracy of refractive prediction determined by different intraocular lens power calculating formulas in hyperopes- A prospective study
}

\author{
Savitha D. P. ${ }^{1}$, Lakshmi B.R. ${ }^{2}$ \\ ${ }^{1}$ Dr. Savitha D. P., ${ }^{2}$ Dr. Lakshmi B.R.; ${ }^{1,2}$ Senior Resident, Department of Ophthalmology, Karnataka Institute of Medical \\ Sciences, Hubli, Karnataka, India.
}

Corresponding Author: Dr. Lakshmi B.R., Senior Resident, Department of Ophthalmology, Karnataka, Institute of Medical Sciences, Hubli, Karnataka, India. Email: lakshmibr24@gmail.com

\begin{abstract}
Purpose: The present study was conducted to evaluate and compare the accuracy of refractive prediction by different intraocular lens power calculating formulas in hyperopes. Methods: This study reviewed 100 eyes of 100 patients who had received cataract extraction with posterior chamber intraocular lens (IOL) implantation using different IOL calculating formulas. The postoperative refractive spherical equivalent (SE)of different IOL calculating formulas (SRK/T, Holladay, Hoffer-Q, and Haigis) was calculated and compared. The SE at different axial lengths (AL) were compared and percentage of postoperative SE value for each formula was calculated at $\pm 0.5 \mathrm{D}$ and $\pm 1.00 \mathrm{D}$. Results: Among the 100 eyes analysed, it was found that haigis formula had the lowest postoperative refractive SE, followed by hoffer-Q and holladay. SRK/T had highest SE. Postoperative SE was positively correlated with AL. Conclusion: Haigis formula rendered the lowest predictive postoperative refractive error compared with holladay, hoffer-Q and SRK/T. Thus, haiges formula may be regarded as a more reliable formula for hyperopes.
\end{abstract}

Keywords: Axial length, Hypermetropes, Intraocular lens power calculating formulas, Spherical equivalent

\section{Introduction}

Obtaining accuracy in intraocular lens power (IOL) calculation is relatively easy in eyes with an axial length between 22 and $26 \mathrm{~mm}$ with normal range (unoperated) corneas. However, the problems that have arisen over the years have been in eyes that are very short $(<22 \mathrm{~mm})$ or very long $(>26 \mathrm{~mm})$. The major problem with short eyes is due to the higher optical power of the required IOL that gives more weight to any error in the predicted IOL position [1].

Over the past several decades there have been many publications showing different results with different formulas. In 1996, Holladay debuted the unpublished Holladay 2 formula, which uses 7 biometric variables and was designed to get the best accu-racy in all ranges of AL. There are few published studies reporting the results with the Holladay 2, however, Hoffer showed in 2000 that the Holladay 2 was equally as accurate as the Hoffer Q in eyes shorter than $22 \mathrm{~mm}$, and that it was less accurate than the Holladay 1 in eyes between 22 and $26 \mathrm{~mm}$ [1]. In 2000, Haigis originated his formula using $\mathrm{AL}$ and the pre-operatively measured ACD to predict the IOL position based on 3 constants. Many studies have shown excellent accuracy of the Haigis formula in all ranges of eyes,

Manuscript received: $20^{\text {th }}$ October 2019

Reviewed: $30^{\text {th }}$ October 2019

Author Corrected: $8^{\text {th }}$ November 2019

Accepted for Publication: $16^{\text {th }}$ November 2019 including short eyes. Eom et al showed that the Haigis formula becomes more accurate than the Hoffer Q in short eyes as the ACD gets shallower than $2.40 \mathrm{~mm}$ [1]. Accurate intraocular lens (IOL) Power calculation in cataract surgery is very important to achieve the postoperative target refraction and high patient satisfaction, as patient expectations have been progressively increased. The refractive power of the human eye depends on the power of the cornea, the lens, the Axial length (AL) of the eye and Axial position of the lens. It has been considered that IOL calculation formulas (SRK/T, Hoffer Q, Holladay 1, and Haigis) were more accurate for eyes with normal AL, but do not have the same level of postoperative refraction outcome for eyes with short AL [1].

Therefore, there has been an ongoing effort to predict the postoperative refractive outcome with accuracy and consistency. The refractive power of the human eye depends on the power of the cornea, the lens, the Axial length (AL) of the eye and Axial position of the lens [2]. There are two main components of the biometry process: Measuring the axial length which can be done by various techniques such as ultrasound A-scan or Immersion method, non-contact by using laser interferometry (IOL Master). Corneal curvature measurement which can be done by keratometer or corneal topography [3]. 


\section{Original Research Article}

IOL power is predicted preoperatively by means of several formulas [2]. Third generation formulas; such as Holladay 1, Hoffer Q and SRK/T; attempt to predict the estimated lens power using AL, corneal curvature $(\mathrm{K})$, and "a" constant as only variables.

Fourth generation formulas, such as Haigis, take into account the preoperative anterior chamber depth (ACD) and uses three constants ( $\mathrm{a} 0, \mathrm{a} 1$, and $\mathrm{a} 2$ ), which are analogous to the surgeon factor (SF), ACD and AL respectively [4]. Of note, inaccuracy in measurement of $\mathrm{ACD}, \mathrm{AL}$ and corneal curvature $(\mathrm{K})$ can contribute to $42 \%, 36 \%, 22 \%$ of errors, respectively [5].

The most commonly applied IOL power formulas today are those by Sanders, Rrtzlaff and Kraff: SRK2 and SRK/T. According to a recent survey in the UK, 49\% used SRK/T, $17 \%$ SRK2 and $34 \%$ other formulas. In Germany, the respective percentages are $40 \%$ for SRK2, $22 \%$ for SRK/T, $23 \%$ for haigis and $15 \%$ for other formulas [6].

In light of the above, the present study aims to evaluate and compare the predictive capacity of four IOL power calculation formulas (SRK/T, Hoffer Q, Holladay 1, and haigis) in eyes with axial length less than $22.00 \mathrm{~mm}$. The present study also evaluated the impact of Axial length (AL), Anterior chamber depth (ACD) and Corneal power(K) on predictability of intraocular lens (IOL) power calculation [6].

\section{Materials and Methods}

Sample size: The study was conducted on 100 eyes from 100 patients attending the outpatient department of Ophthalmology

Sampling method: Random table number

Study duration: November 2015 to August 2017

Inclusion criteria:

1. Axial length $(\mathrm{AL})<22.00 \mathrm{~mm}$.

2. Presence of cataract.

\section{Exclusion criteria:}

Factors likely to affect biometry readings, affect IOL positioning in the capsular bag or decrease the accuracy of measured refraction.

\section{Abnormalities of cornea}

2. Previous intraocular or corneal surgery (including kerato refractive surgery)

3. History of ocular injury or uveitis

4. Intraoperative complications such as posterior capsule rupture, vitreous loss, lost nucleus, zonular dehiscence, and wound leak.

Ethical clearance: Study is approved by ethical committee

Data collection - After detailed history, complete ocular examination including visual acuity examination, dilated refraction, slit lamp examination, intraocular pressure measurement, fundoscopy was done. Biometry was done using Automated keratometry and A-scan USG machine (EPIDOT USO), IOL power was calculated using four IOL calculating formulas (Hoffer Q, Holladay1, SRK2 and Haigis). 100 patients are divided into 4 groups randomly, were each group contains 25 patients as Hoffer Q group, Holladay1 group, SRK 2 group and Haigis group. Small incision cataract surgery is done to all patients with posterior chamber IOL power calculated using any one of the above formulae. Postoperative Dayl vision and refraction, 1-month postoperative vision and refraction was recorded.

Statistical Analysis - performed using IBM statistical package for social sciences software (SPSS) Version - 17 for Windows. Results are presented as mean $\pm \mathrm{SD}$ (standard deviation), Numbers and Percentages. One-way ANOVA Test was used for multiple group comparison followed by Post-HOC- TUKEY'S Test for group wise comparison. Unpaired T- Test was used to compare Mean values between the 2 groups. Categorical data was analysed by chisquare Test for the association of 2 factors. Pearson's CoRelation Co-Efficient was used to assess the relation between 2 measurements.

\section{Results}

In the current study, 9 patients had pre-senile cataract and remaining 91 patients has senile cataract. The study included 39 males and 61 females. The age of the patients ranged from 34 to 88 years, with mean age being $62.59 \pm 11.29$ years.

On comparison of average K(D) and Axial length (mm) among the 4 groups using one Way ANOVA test, p- value of 1.15 and 0.73 were obtained, suggesting a statistically no significant correlation. But comparison of ACD among 4 groups shows p- value of 0.02 , suggesting statistically significant correlation. Comparison of average $K(D)$ and Axial length among 4 groups, showed statistically no significant correlation.

Group wise comparison of IOL powers obtained by different formulas, showed p-value $<0.05$ in all 4 study groups, suggesting statistically no significant correlation. Group wise comparison of IOL powers obtained by different formulas, showed p-value $<0.05$ in all 4 study groups, suggesting statistically no significant correlation (Table 1). 
Original Research Article

Table-1: Comparison of various parameters among 4 study groups.

\begin{tabular}{|l|c|c|c|c|c|c|c|}
\hline Groups & Av K (D) & AXL (mm) & ACD (mm) & SRK2 & HOLLA & HOFFQ & HAIGS \\
\hline Gr 1 & $44.78 \pm 2.47$ & $21.42 \pm 0.56$ & $3.51 \pm 0.54$ & $26.06 \pm 2.26$ & $25.70 \pm 2.26$ & $25.58 \pm .2 .11$ & $25.28 \pm 2.20$ \\
\hline Gr 2 & $45.23 \pm 2.67$ & $21.39 \pm 0.53$ & $3.47 \pm 0.58$ & $26.58 \pm 2.33$ & $26.22 \pm .22$ & $26.16 \pm 2.1$ & $25.86 \pm 2.29$ \\
\hline Gr 3 & $43.78 \pm 1.93$ & $21.36 \pm 0.36$ & $3.11 \pm 0.42$ & $25.82 \pm 1.12$ & $25.66 \pm 1.21$ & $25.46 \pm 1.17$ & $25.08 \pm 1.07$ \\
\hline Gr 4 & $44.18 \pm 2.45$ & $21.27 \pm 0.54$ & $3.44 \pm 0.46$ & $26.38 \pm 1.96$ & $26.02 \pm 2.00$ & $25.88 \pm 1.99$ & $25.70 \pm 1.89$ \\
\hline Anova F & 1.81 & 0.43 & 3.30 & 0.73 & 0.46 & 0.67 & 0.88 \\
\hline P value & 0.15 & 0.73 & $0.02 *$ & 0.54 & 0.71 & 0.57 & 0.45 \\
\hline
\end{tabular}

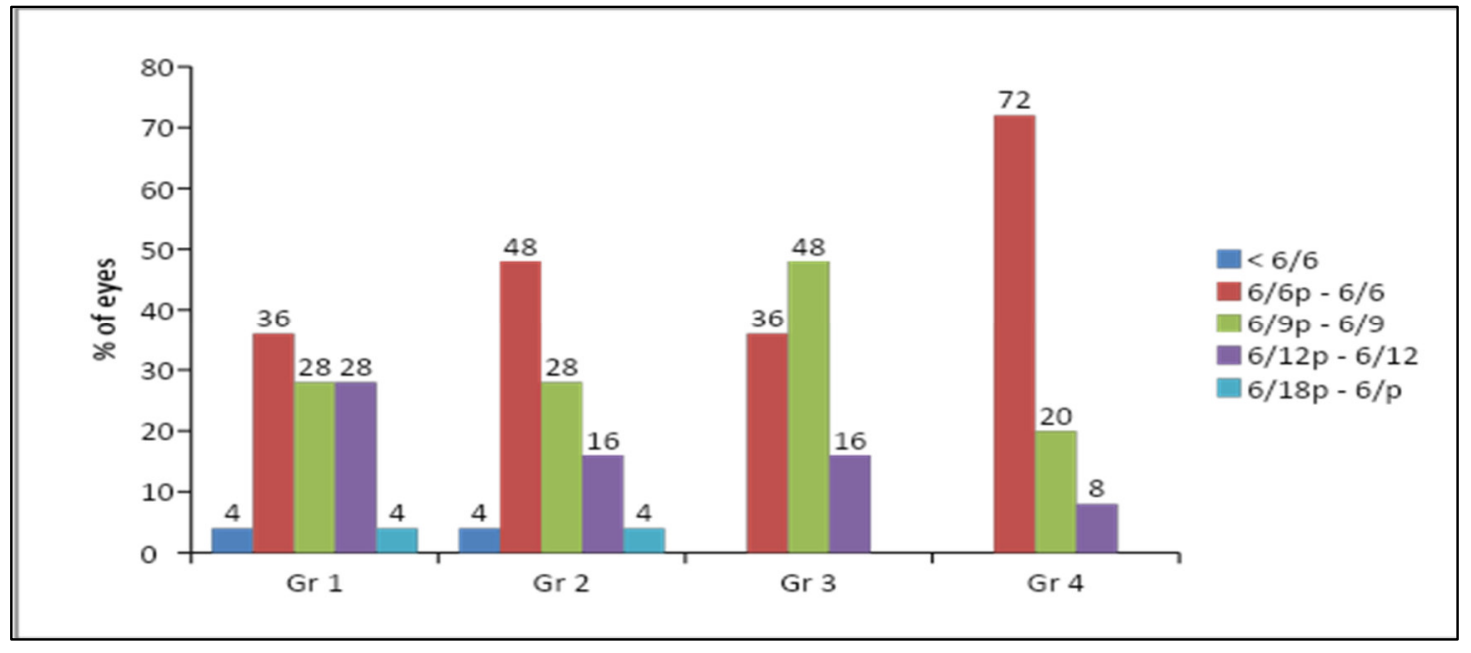

Fig-1: Day 1 postoperative VA comparison among 4 groups.

Among 4 groups, Haigis group showed $72 \%$ of immediate postoperative VA ranging between 6/6p-6/6, compared to $36 \%$, $48 \%$ and $36 \%$ in group 1, 2 and 3 respectively (Figure 1).

\section{Month postoperative visual acuity}

Table-2: One-month post-op (M1) Visual Acuity.

\begin{tabular}{|c|c|c|c|c|}
\hline \multirow{2}{*}{ V A } & Group 1 & Group 2 & Group 3 & Group 4 \\
\cline { 2 - 5 } & $\mathbf{n ~ ( \% )}$ & $\mathbf{n ~ ( \% )}$ & $\mathbf{n}(\%)$ & n (\%) \\
\hline$<6 / 6$ & - & - & - & - \\
\hline $6 / 6 p-6 / 6$ & $5(20)$ & $5(20)$ & $12(48)$ & $16(64)$ \\
\hline $6 / 9 p-6 / 9$ & $13(52)$ & $17(68)$ & $5(20)$ & $4(16)$ \\
\hline $6 / 12 p-6 / 12$ & $5(20)$ & $3(12)$ & $0(0)$ & $0(0)$ \\
\hline $6 / 18 p-6 / p$ & $2(8)$ & $0(0)$ & $\mathbf{2 5}(\mathbf{1 0 0})$ & $\mathbf{2 5}(\mathbf{1 0 0})$ \\
\hline Total & $\mathbf{2 5 ( 1 0 0 )}$ & $\mathbf{2 5 ( 1 0 0 )}$ & \\
\hline
\end{tabular}

Group wise comparison of 1-month postoperative VA using Chi-square test, showed 20\%, 20\%, 32\% and 20\% of patients had 6/6p- 6/6 VA in group 1,2,3 and 4 respectively. Table 2 comparison of 1-month postoperative VA among 4 groups.

Group wise comparison of 1-month postoperative VA showed $68 \%$ of cases with VA between $6 / 9 \mathrm{p}-6 / 9$ in group 2 compared to $52 \%, 48 \%$ and $64 \%$ among group 1,3 and 4 respectively. 
Original Research Article

Table-3: Pre and Post-operative Spherical Equivalent among 4 study groups.

\begin{tabular}{|c|c|c|c|c|}
\hline \multicolumn{2}{|l|}{ Groups } & Pre SE & D1 SE & M1 SE \\
\hline \multicolumn{2}{|l|}{ Gr 1} & $1.02 \pm 1.22$ & $0.50 \pm 0.35$ & $0.64 \pm 0.32$ \\
\hline \multicolumn{2}{|l|}{ Gr 2} & $0.91 \pm 1.31$ & $0.54 \pm 0.42$ & $0.53 \pm 0.29$ \\
\hline \multicolumn{2}{|l|}{ Gr 3} & $1.22 \pm 1.39$ & $0.59 \pm 0.22$ & $0.49 \pm 0.20$ \\
\hline \multicolumn{2}{|l|}{ Gr 4} & $1.55 \pm 1.63$ & $0.31 \pm 0.10$ & $0.30 \pm 0.15$ \\
\hline \multicolumn{2}{|l|}{ ANOVA F } & 0.99 & 4.07 & 8.01 \\
\hline \multicolumn{2}{|l|}{ P value } & $0.40, \mathrm{~ns}$ & $0.01 *$ & $0.00 * *$ \\
\hline \multirow{6}{*}{$\begin{array}{l}\text { Difference between } \\
\text { groups } \\
\text { (P values) }\end{array}$} & $1-2$ & NS & 0.95 & 0.46 \\
\hline & $1-3$ & NS & 0.93 & 0.15 \\
\hline & $1-4$ & NS & 0.10 & $0.00^{* *}$ \\
\hline & $2-3$ & NS & 0.96 & 0.91 \\
\hline & $2-4$ & NS & $0.03 *$ & $0.01^{*}$ \\
\hline & $3-4$ & NS & $0.01 *$ & $0.04 *$ \\
\hline
\end{tabular}

Group wise comparison of pre and postoperative comparison among 4 groups using One Way ANOVA test showed $p$ value of 0.00 suggestive of statistically highly significant correlation between group 1 and 4 in 1 -month post-operative SE. And P-value of 0.03 and 0.01 seen between group $2-4$ and group 3-4 respectively in Day 1 postoperative SE, suggesting significant statistical correlation. Also, $\mathrm{p}$ - value 0.01 and 0.04 seen between group 2-4 and 3-4 respectively in 1-month postoperative SE, suggesting significant statistical correlation (Table 3). Among the study groups, group 4 showed least postoperative SE of 0.31 (D) compared to $0.59,0.54$ and 0.5 (D) in group 1,2 and 3 respectively.

Table-4: Percentage of eyes within specified target refraction for each group

\begin{tabular}{|c|c|c|c|c|}
\hline \multirow{3}{*}{ Groups } & \multicolumn{2}{|c|}{ Day 1} & \multicolumn{2}{|c|}{1 month } \\
\hline & $\pm 0.5 \mathrm{D}$ & $\pm 1.0 \mathrm{D}$ & $\pm 0.5 \mathrm{D}$ & $\pm 1.0 \mathrm{D}$ \\
\hline & n (\%) & n (\%) & n (\%) & n (\%) \\
\hline Gr 1 & $17(68)$ & $23(92)$ & $13(52)$ & $22(88)$ \\
\hline Gr 2 & $16(64)$ & $21(84)$ & $15(60)$ & $24(96)$ \\
\hline Gr 3 & $12(48)$ & $25(100)$ & $16(64)$ & $25(100)$ \\
\hline Gr 4 & $24(96)$ & $25(100)$ & $25(100)$ & $25(100)$ \\
\hline
\end{tabular}

Group wise comparison of percentage of eyes with specified target refraction at immediate postoperatively, showed $96 \%$ cases were within $\pm 0.5 \mathrm{D}$ in group 4 compared to $68 \%, 64 \%$ and $48 \%$ in group 1,2 and 3 respectively. $100 \%$ cases were within $\pm 1.00 \mathrm{D}$ of targeted refraction at Day 1 postoperatively in group 3 and 4 compared to $92 \%$ and $84 \%$ in group 1 and 2 respectively. $100 \%$ cases from group 4 showed targeted refraction within $\pm 0.5 \mathrm{D}$ at 1 month postoperatively compared to $52 \%, 60 \%$ and $64 \%$ in group 1,2 and 3 respectively. $100 \%$ cases were within $\pm 1.00 \mathrm{D}$ of targeted refraction at 1 month postoperatively in group 3 and 4 compared to $88 \%$ and $96 \%$ in group 1 and 2 respectively (Table 4).

\section{Discussion}

In this study comparison between the accuracies of four widely used IOL power calculating formulas, namely, SRK/T, Holladay, Hoffer-Q and Haigis applying for 100 hyperopic eyes was made. The significance of this study is to reduce the postoperative refractive prediction error, which gradually affects patients prognosis. In addition, this is one of the few studies supportive of the fact that the use of Haigis formula would enhance the refractive predictability in hyperopes.
Of all the components required to determine IOL power, inaccurate measurement of the axial length (AL) of the eyes is the most frequent factor causing unexpected outcomes.

An AL measurement that is erroneous by $100 \mu \mathrm{m}$ translates into $0.28 \mathrm{D}$ error in the postoperative refraction. Though inaccurate corneal power measurements account for a much smaller percentage of unexpected outcomes, careful attention should always be paid to keratometry [7]. 
Original Research Article

similar results were obtained in other studies conducted by Marilita M. Moschos [2] who reported Haigis formula had statistically significant smaller mean $\mathrm{AE}$ in comparison to Holladay 1, Hoffer Q and SRK/T. Wang JK et.al reported Haigis formula yields superior refractive results ineyes with various axial length using IOL Master.

In the present study, it was found Haigis group had showed least postoperative SE, indicating that Haigis formula is the more accurate than Hoffer Q, Holladay 1 and SRK2 in predicting the postoperative refraction after cataract surgery in eyes with axial length $<22.00 \mathrm{~mm}$, which is similar to the observations of Marilita M. Moschos [8], Oslen T[9], Thim K [10], Alexander C. LEE [11], Mujtaba A [12].

However, EA Gavin and CJ Hammond [13] in contrast to the present study, Hoffer Q was found to be more accurate than the SRK-T formula in eyes $<22 \mathrm{~mm}$ axial length.

In Contrast, predictive accuracy of various IOL power calculation formulas in eyes with short AL. Narvaez et al [14] employed immersion ultrasonography and manual keratometry to evaluate 25 eyes with AL less than $22.0 \mathrm{~mm}$, suggesting no statistically significant difference between Holladay 1, Holladay 2, Hoffer Q, and SRK/T.

Gavin and Hammond [15] investigated 41 eyes with AL less than $22 \mathrm{~mm}$, measured by IOL Master, concluding that the Hoffer Q formula was more accurate than the SRK/T.

A potential limitation of the present study pertains to the fact that

1. Partial coherence interferometry method (IOL Master) was not used, which is considered as the most accurate method in IOL calculation

2. Applanation A-scan method was used instead Immersion method, which could be a potential source of error in Axial length calculation.

\section{Conclusion}

In conclusion, the present study suggested that Haigis formula provides more accurate results concerning the postoperative targeted refraction in eyes with axial length less than $22.00 \mathrm{~mm}$. Hoffer $\mathrm{Q}$ could be used as an alternative. multiple studies with larger population are needed to assess the efficacy and importance of this study.

\section{What this study adds to existing knowledge?}

The present study aims to evaluate and compare the predictive capacity of four IOL power calculation formulas (SRK/T, Hoffer Q, Holladay 1, and haigis) in eyes with axial length less than $22.00 \mathrm{~mm}$. The present study also evaluated the impact of Axial length (AL), Anterior chamber depth (ACD) and Corneal power (K) on predictability of intraocular lens (IOL) power calculation.

\section{Author's contribution}

Dr. Savitha D. P.: Concept, study design, manuscript preparation

Dr. Lakshmi B.R: Concept, statistical analysis, manuscript preparation

Funding: No funding sources

Conflict of interest: None declared

Ethical Approval: This study was approved by the Institutional Ethics Committee

\section{Reference}

1. Hoffer KJ, Savini G. IOL power calculation in short and long eyes. Asia-Pac J Ophthalmol. 2017;6(4):330-331. doi: 10.22608/APO.2017338.

2. Moschos MM, Chatziralli IP, Koutsandrea C. Intraocular lens power calculation in eyes with short axial length. Ind $\mathrm{J}$ Ophthalmol. 2014;62(6):692-694. doi: 10.4103/0301-4738. 129791.

3. B. Vineeth Kumar (UK), Mastering the techniques of IOL power calculation $2^{\text {nd }}$ edition, 121.

4. Oslen T, Thim K, Corydon L. Accuracy of newer generation intraocular lens power calculation formulas in long and short eyes. J Cat Refract Surg. 1991;17(2):187193. doi: https://doi.org/10.1016/S0886-3350(13)80249-0.

5. Lee AC, Qazi MA, Pepose JS. Biometry and intraocular lens power calculation. Curr Opinion Ophthalmol. 2008; 19(1):13-17

6. Wolfgang Haigis (germany), IOL Calculation in Long and Short eyes, Mastering the techniques of IOL power calculation, $2^{\text {nd }}$ edition, 115 .

7. Noel Alpins, FR Cophth, FACS and Gemma Walsh, B Optom. Accurate biometry and IOL calculation chapter 24, page 237.

8. Rhonda G Waldron, MMSc, COMT, CRA, ROUB. AScan Biometry updated; Apr 20, 2016.

9. Nemeth G, Vajas A, Kolozsvari B, Berta A, Modis Jr L. Anterior chamber depth measurements in phakic and pseudophakic eyes: Pentacam versus ultrasound device. J Cataract Refract Surg. 2006;32(8):1331-1335. doi: https:// doi. org/10.1016/j.jcrs.2006.02.057. 


\section{Original Research Article}

10. Hitzenberger CK, Drexler W, Dolezal C, Skorpik F, Juchem M, Fercher AF, et al. Measurement of the axial length of cataract eyes by laser Doppler interferometry. Invest Ophthalmol Vis Sci. 1993;34(6):1886-1893.

11. Kiss B, Findl O, Menapace R, Wirtitsch M, Petternel V, Drexler W, et al. Refractive outcome of cataract surgery using partial coherence interferometry and ultrasound biometry: clinical feasibility study of a commercial prototype II. J Cataract Refract Surg. 2002;28(2):230-234. doi: https://doi.org/10.1016/S0886-3350(01)01274-3.

12. Olsen T. Improved accuracy of intraocular lens power calculation with the Zeiss IOL Master. Acta Ophthalmol Scand. 2007; 85(1):84-87. doi: https://doi.org/10.1111/j. 1600-0420. 2006.00774.x.
13. JT Lin, A Garg; An update on IOL power calculation Formulas, Mastering the techniques of IOL power calculation, chapter $11,2^{\text {nd }}$ edition, page 51-61.

14. Narváez J, Zimmerman G, Stulting RD, Chang DH. Accuracy of intraocular lens power prediction using the Hoffer Q, Holladay 1, Holladay 2, and SRK/T formulas. J Cataract Refract Surg. 2006;32(12):2050-2053. doi: https:// doi. org/10.1016/j.jcrs.2006.09.009.

15. Gavin EA, Hammond CJ. Intraocular lens power calculation in short eyes. Eye. 2008;22(7):935-938. doi: https://doi.org/10.1038/sj.eye.6702774.

\section{How to cite this article?}

Savitha D. P, Lakshmi B.R. Accuracy of refractive prediction determined by different intraocular lens power calculating formulas in hyperopes- A prospective study. Trop J Ophthalmol Otolaryngol.2019;4(7):440-445.doi:10.17511/jooo.2019.i07.07 